Monatsschrift für Geburtshülfe u. Gynäkologie 1910;32:I-VI

\title{
Contents, Vol. 32, 1910
}

\section{Original-Arbeiten.}

A d 1 e r , L., Zur Klinik der Retroversioflexio uteri .... 298 Amersbach, K., Eitriger Katarrh der Tube nach Ein-

leitung des künstlichen Aborts

Aschner, Bernhard, Ueber einen Fall von hypo-

plastisehem Zwergwuchs mit Gravidität nebst Bemerkungen über die Aetiologie des

Zwergwuchses.

(Hierzu Taf. V-VI) 641

Bien, Gertrud, Ueber Furchenbildung an der Ober-

fläche des menschlichen Ovariums. (Hierzu Taf. II). . 175

Cramer, H., Varikocele graviditatis 551

, Pseudomyxoma peritonei nach Appendicitis

Dahlmann, Albert, Zerreissungen der Gebärmutter

nach Kaiserschnitt 33

Fetzer, Max, Zur Klinik der Insertio velamentosa funi-

culi ximbilicalis 23

Frank, Ed., Zur Lehre der Tetania gravidarum .... 416 Frank, Fritz, Ueber den subkutanen

Symphysen-

schnitt und die suprasymphysäre Entbindung .... 680 Frank el, L., Rückbildung von

Ovarialtumoren nach

Blasenmole 180

Franz, K., Operative Myombehandlung 153

Franz, R., Ueber Virulenzbestimmung der Streptokokken

mittels Lecithinbouillon 287

From me, F., Einige Bemerkungen zur Lehre von der

intrauterinen Atmung, zur Händedesinfektion und zur

Frage der Selbstinfektion $\quad 555$

Graff, Erwin v., Zum Nachweis hämolytischer Stoffe

in der Plazenta 125

Gussakow, L., Kritische Beurteilung der gegenwärtigen

Ansichten über Plazenta praevia 245

Haase, Felix, Beitrag zur Kenntnis der Entstehungs-

ursachen amniotischer Strange

Heimann, Fritz, Klinisches zur Frage der Polydaktylie

(Hierzu Taf. I) 144

Hofstätter, Robert, Die Behandlung der post-

partum-Blutungen 470

Hoehne, 0., Ueber die Operabilität der Rezidive nach

Uteruskarzinomoperationen 161 
Holzbach, E., Zur Genese kombinierter Nieren-Uterus-

Blutungen. (Hierzu Taf. V) 406

IV -

Jacobson, W. L., Die vaginale Koeliotomie bei ek-

topischer Schwangerschaft (Ergänzungs-Heft) .... 1

Kaji, Zur ovariellen Aetiologie uteriner Blutungen ... 427

Kaufman, Mieczyslaw, Ueber mehrmalige Zer-

reissungen des Scheidengewölbes während der Geburt 148

Kiparsky, Rene, Der vaginale Weg in der operativen

Behandlung der Uterusgeschwülste (Ergänzungs-Heft) 8

Korobkoff, A., Die vaginale Ovariotomie während der

Schwangerschaft (Ergänzungs-Heft) 13

Kuhn, Franz, Steril-Kalgut 710

Küstner, O., Abdominale Totalexstirptaion des Uterus, Ventrifixur des Scheidengewölbes, Kolporrhaphien; Ver-

fahren bei grossen Prolapsen

1

Latzko, W., Die Levatornaht als tvpische Prolapsoperation

(Hierzu Taf. Ill-IV) ... 330

M a r k o w s k y , A., Zur Erage der Behandlung der Adnexerkrankungenmittels Coeliotomia vaginalis (Ergänz.-Heft) 18

Martin, A., Geburtshülfe und Gynäkologie im ersten

Jahrhundert der Berliner Hochschule 1810-1910. 385, 590

M a r t i n , E d., Ein Modell des weiblichen Beckens .... 587

Mohr, Ludwig, Ueber einen Fall von Spaltbecken mit

Ectopia vesicae totalis

3

Novak, J. , und L. J e 11 e r , Beitrag zur Kenntnis der

puerperalen Bradykardie 531

O 1 o w , John, Zwei Fälle von Achsendrehung des Uterus . 53

O 11 , D. v., Wodurch werden die besseren Erfolge bei den vaginalen Coeliotomien im

Vergleich mit denjenigen bei abdominalen bedingt ? (Ergänzungs-Heft) .......

Pankow, Otto, Zur Frage der kongenitalen Ueber-

tragung der Tuberkulose 579

Peters, H., Ein seltener polypöser Anhang der Vaginal-

portion 573

Preobrajensky, W., Ueber den Konservatismus bei

Koeliotomien (Ergänzungs-Heft) 25

Puppel, Ernst, Erwiderung auf den Artikel Rosensteins: ,, Ueber die Behandlung der anderen

nicht graviden Tube bei Operation wegen Tubargravidität . 731

Rimann, H, Zur Lehre von der Tympania uteri .... 673

Rosenstein, M., Wie ist bei der Operation einer Tubargravidität die Tube der anderen Seite zu behandeln ? 435

, Erwiderung auf die Bemerkungen des Herrn Puppel 732

Sachs, E., Status epilepticus und Schwangerschaft . . 649

Schaback, Karl F., Ueber Primär- und Dauerresultate

bei kompJeten Dammriss-Operationen 
Schaeffer, Rich. Plastische Modelle zur Grössenbestimmung von Unterleibstiimoren, inbesondere der schwangeren Gebärmutter 463

Schiffmann, Josef, und Rudolf Ekler, Die

ätiologische Therapie des Prolapses 335

Schweninger, Franz, Frühaufstehen Operierter und Thrombophlebitis 11

V -

Sellheim, $\mathrm{H} \backslash_{\imath} \mathrm{g}$ o , Verwertung der bei der Operation

gebräuchlichen temporären Abkammerung der freien

Bauchhöhle zur Beschränkung einer im freien Operations-

gebiete einsetzenden Infektion während der Nach-

behandlungsperiode 693

Sitzenfrey, Anton, Ueber Saugbehandlung der

puerperalen Endometritis 261

Sitzinsky , A., Ueber dieBeleuchtung der Höhlé des puerperalen Uterus. (Ergänzungs-Heft.)

(Hierzu Taf. VII) 51

Soboloff, Jean de, Die Appendicektomie als be-

gleitende Operation bei vaginalen Koeliotonúen. (Er

gänzungs-Heft) $\quad 46$

Sonnenfeld, Julius, Zur kombinierten Unter-

suchung $\quad 572$

Sticker, Anton, und Edmund E a 1 k, Die Be-handlung des inoperablen Krebses mit radio-

aktiven Eermenten- Carboradiogen und Carboradiogenol. . . 703

Theilhaber, A., Der Zusammenhang von My omen

mit internen Erkrankungen 455

Vértes, Oskar, Ueber das wechselseitige Verhältnis der

weiblichen Geschlechtsorgane und der Erkrankungen des

Magens 128

W e r b o f f, J., Der vaginale Weg bei der Komplikation von

Schwangerschaft mit Carcinoma uteri. (Ergänzungs-Heft) 48

Sammelreferate.

Aus der serologischen Literatur. $\lambda$-"on Dr. OskarErankl

in Wien 591

Zur Anästhesierungsfrage in der Gynäkologie. (Lokal- und

Lumbalanästhesie.) Von Dr. G. A. Wagner in Wien 712

Bericht über die amerikanische geburtshülflich-gynäkologische

Literatur. II. Halbjahr 1908. Von Dr. ArthurStein

in New York 67

Bericht über die französische geburtshülflich-gynäkologische

Literatur. Von Prof. Dr. CyrilleJeanninin Paris 76

Die geburtshülfliche und gynäkologische Literatur in Holland.

I. Halbjahr 1909. Von Dr. M. M. de Monchy im

Haag 63

Berichte. 
8. Versammlung des Nordischen chirurgischen Vereins in Helsingfors vom 19.-21. August 1909. Bericht, erstattet von Dr. V. A 1 b e c k in Kopenhagen $\quad 80$

Die geburtshülflich-gynäkologische Sektion der 82. Naturforscher - Versammlung in Königsberg vom 18. bis

22. September 1910. Von A. Martin 484

$\mathrm{P}^{1} / 8$ eferat über die Abteilung XVIII fur Geburtshülfe und Gynäkologie der 82. Versammlung deutscher Natur-

$-\mathrm{VI}-$

forscher und Aerzte in Königsberg i. Pr. September

1910, erstattet von Prof. Dr. Hammerschlag in

Berlin 480̃

Der V. Internationale Kongress für Geburtshülfe und

Gynäkologie. Von A. Martin 241

St. Petersburg, 22.-27. September 1910. Von A. M a r t i n 613

Verhandlungen des V. Internationalen Gynäkologen-Kon-

gresses in St. Petersburg vom 22.-27. September 1910.

Bericht, erstattet von Dr. W. Beckmann in

St. Petersburg . : 616

Die Centenarfeier der Universitäts-Frauenklinik zu Leipzig

(Triersches Institut) am 8. Oktober 1910. Von Prof.

Dr. H. F ü t h in Köln 610

RudolfChrobak†. (Mit Porträt)

517

Tagesnach $\Gamma$ ichten und Personalien 185, 240, 357, 360, 493, 634, 733

Vereins- und Literaturbeilage.

Gynäkologische Gesellschaft in Breslau $\quad 86,186, \quad 361$

Gesellschaft für Geburtshülfe und Gynäkologie zu Köln . . 214

Mittelrheinische Gesellschaft für Geburtshülfe und Gynä

kologie . 369

Münchener gynäkologische Gesellschaft $93,494, \quad 734$

Mederrheinisch-westfälische Gesellschaft für Gynäkologie

und Geburtshülfe 104, 209

Nordwestdeutsche Gesellschaft für Gynäkologie

Russische Gesellschaft für Geburtshülfe und Gynäkologie 222

Referate aus geburtshülflich-gynäkologischen Gesellschaften

$117,230,378,509,635, \quad 742$

Geburtshülflich-gynäkologische Mitteilungen aus anderen $\mathrm{Ge}$

sellschaften und Vereinen 118, 232, 379, 510, 636, 742

Buchanzeigen 119, 233, 380, 510, 743

Literatur-Verzeichnis 121, 235, 381, 513, 636, 73

Sachregister

747

Namenregister

755 